\title{
Universal inhomogeneous magnetic-field response in the normal state of cuprate high- $T_{c}$ superconductors
}

\author{
Z. Lotfi Mahyari and A. Cannell \\ Department of Physics, Simon Fraser University, Burnaby, British Columbia, Canada V5A 1 S6 \\ E.V.L. de Melld* \\ Instituto de Física, Universidade Federal Fluminense, Niterói, RJ 24210-340, Brazil \\ M. Ishikado \\ Research Center for Neutron Science and Technology, Tokai, Naka, Ibaraki, Japan 319-1106 \\ H. Eisaki \\ National Institute of Advanced Industrial Science and Technology, Tsukuba, Ibaraki, Japan 305-8568 \\ Ruixing Liang and D.A. Bonn \\ Department of Physics and Astronomy, University of British Columbia, \\ Vancouver, British Columbia, Canada V6T $1 Z 1$ and \\ Canadian Institute for Advanced Research, Toronto, Canada M5G $1 Z 8$ \\ J.E. Sonier \\ Department of Physics, Simon Fraser University, \\ Burnaby, British Columbia, Canada V5A 1 S6 and \\ Canadian Institute for Advanced Research, Toronto, Canada M5G $1 Z 8$
}

(Dated: July 21, 2018)

\begin{abstract}
We report the results of a muon spin rotation ( $\mu \mathrm{SR}$ ) study of the bulk of $\mathrm{Bi}_{2+x} \mathrm{Sr}_{2-x} \mathrm{CaCu}_{2} \mathrm{O}_{8+\delta}$, as well as pure and $\mathrm{Ca}$-doped $\mathrm{YBa}_{2} \mathrm{Cu}_{3} \mathrm{O}_{y}$, which together with prior measurements reveal a universal inhomogeneous magnetic-field response of hole-doped cuprates extending to temperatures far above the critical temperature $\left(T_{c}\right)$. The primary features of our data are incompatible with the spatially inhomogeneous response being dominated by known charge density wave (CDW) and spin density wave (SDW) orders. Instead the normal-state inhomogeneous line broadening is found to scale with the maximum value $T_{c}^{\max }$ for each cuprate family, indicating it is controlled by the same energy scale as $T_{c}$. Since the degree of chemical disorder varies widely among the cuprates we have measured, the observed scaling constitutes evidence for an intrinsic electronic tendency toward inhomogeneity above $T_{c}$.

PACS numbers: 74.72.-h, 74.25.Ha, 76.75.+i
\end{abstract}

\section{INTRODUCTION}

Experiments probing the normal state of high- $T_{c}$ cuprate superconductors have provided evidence for the presence of electronic nematicity,$\frac{1.2}{,}$ fluctuating stripes,$\frac{3}{\underline{-}}$

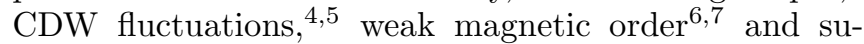
perconducting fluctuations (SCFs) or phase-incoherent Cooper pairs ${ }^{8-13}$ At present there is a quest for commonality amongst these findings, and debate over the temperature range of SCFs above $T_{c}$.

The initial discovery of a vortex-motion contribution to the Nernst signal in the normal state of cuprate superconductors, $\stackrel{9}{=}$ advocated the occurrence of phasefluctuating superconductivity up to temperatures several times $T_{c}$. This finding was subsequently supported by high-resolution torque magnetometry experiments, which detected field-enhanced diamagnetism at equally high temperatures: $\frac{11.12}{}$ More recently, precursor superconductivity persisting up to $180 \mathrm{~K}$ has been inferred from the infrared $c$-axis response of $R \mathrm{Ba}_{2} \mathrm{Cu}_{3} \mathrm{O}_{y}(R=$
Y, Gd, Eu) $\stackrel{13}{ }$ Yet other studies have argued that a noticeable contribution of SCFs to the Nernst response of cuprates is present only at temperatures 10 to $25 \mathrm{~K}$ above $T_{c}, 2,14,15$ A narrow region of SCFs above $T_{c}$ has also been concluded from AC conductivity studies $, \underline{8}, 16,17$

Distinct from these bulk measurements is a scanning tunneling microscopy (STM) study of the surface of $\mathrm{Bi}_{2+x} \mathrm{Sr}_{2-x} \mathrm{CaCu}_{2} \mathrm{O}_{8+\delta}$ (BSCCO), which shows the nucleation of pairing gaps in nanoscale regions persisting to temperatures above the detection of diamagnetism. 10 Although the lack of consensus about the temperature extent of SCFs above $T_{c}$ may be attributed to varying degrees of sensitivity of different techniques to inhomogeneous superconducting correlations, BSCCO, and more so its surface, are highly disordered. Whether the same nanoscale electronic inhomogeneity observed at the surface of BSCCO above $T_{c}$ is also present in the bulk, and whether it has any relevance to other cuprate superconductors are questions of fundamental importance.

Expanding on earlier measurements $\stackrel{18-20}{\underline{2}}$ we have used 
transverse-field (TF) $\mu \mathrm{SR}$ to investigate the situation in the bulk of BSCCO and optimally-doped and Ca-doped $\mathrm{YBa}_{2} \mathrm{Cu}_{3} \mathrm{O}_{y}$ (YBCO) single crystals. The implanted positive muon is a pure local magnetic probe, and relaxation of the time-dependent TF- $\mu \mathrm{SR}$ signal results from a distribution of internal magnetic field $n(B)$. The line width of $n(B)$ and the corresponding relaxation rate are reduced as the internal magnetic field becomes more uniform, but are also diminished by fluctuations of the local field.

\section{EXPERIMENTAL DETAILS}

The samples are all plate-like single crystals. The pure and Ca-doped YBCO single crystals were grown by a selfflux method in fabricated $\mathrm{BaZrO}_{3}$ crucibles, as described elsewhere, $\stackrel{21}{\stackrel{1}{1}}$ and assembled into mosaics of 6 to 10 single crystals from the same growth batch. Typical sample sizes were $5 \times 5 \times 0.1-0.2 \mathrm{~mm}^{3}$. Zero-field (ZF) $\mu \mathrm{SR}$ measurements on some of the YBCO samples are presented in Ref. 22. The BSCCO samples studied are of similar dimensions, and consist of 1 or 2 single crystals grown by the traveling-solvent-floating-zone method. Sample compositions of $\mathrm{Bi}_{2+x} \mathrm{Sr}_{2-x} \mathrm{Ca}_{2} \mathrm{Cu}_{2} \mathrm{O}_{8+\delta}$ with $x=0.1$ to 0.15 were fabricated from powders of $\mathrm{Bi}_{2} \mathrm{O}_{3}, \mathrm{SrCO}_{3}, \mathrm{CaCO}_{3}$, and $\mathrm{CuO}$ as starting materials. After pre-melting the polycrystalline rod, crystal growth was carried out in air and at a feed speed of 0.15 to $0.20 \mathrm{~mm} / \mathrm{h}$ for about 3 weeks. The doping level was adjusted by tuning the excess oxygen content. The underdoped BSCCO sample was annealed at $570{ }^{\circ} \mathrm{C}$ under flowing $\mathrm{N}_{2}$ gas with less than $10 \mathrm{ppm}$ oxygen concentration for $72 \mathrm{hrs}$. Overdoping was achieved by annealing at $400{ }^{\circ} \mathrm{C}$ under an oxygen partial pressure of $2.3 \mathrm{~atm}$ for 72 to $250 \mathrm{hrs}$. The optimally-doped BSCCO sample was annnealed in air at $720{ }^{\circ} \mathrm{C}$ for $24 \mathrm{hrs}$. A superconducting quantum interference device (SQUID) magnetometer was used for measurements of $T_{c}$.

The TF- $\mu$ SR experiments were carried out on the M15 surface muon channel at TRIUMF (located in Vancouver, Canada) using the so-called HiTime spectrometer, which features ultra-low background and high magnetic field capabilities. The magnetic field $\mathbf{H}$ was applied parallel to the $c$ axis of the sample by a $7.0 \mathrm{~T}$ superconducting splitcoil solenoid. Nearly $100 \%$ spin-polarized positive muons were implanted into the sample with the initial muonspin polarization $\mathbf{P}(0)$ transverse to the direction of the applied field (see Fig. 1). The muon magnetic moment undergoes Larmor precession at a frequency proportional to the local internal magnetic field (i.e. $\omega=\gamma_{\mu} B$, where $\gamma_{\mu}=851.6 \mathrm{MHz} / \mathrm{T}$ is the muon gyromagnetic ratio), which is the vector sum of the applied field penetrating the sample, and the dipolar fields associated with the magnetic moments of the host nuclei and electrons. The time evolution of the muon-spin polarization $P(t)$ reflects the distribution of internal magnetic fields experienced by the muon ensemble, and is monitored through the detec-

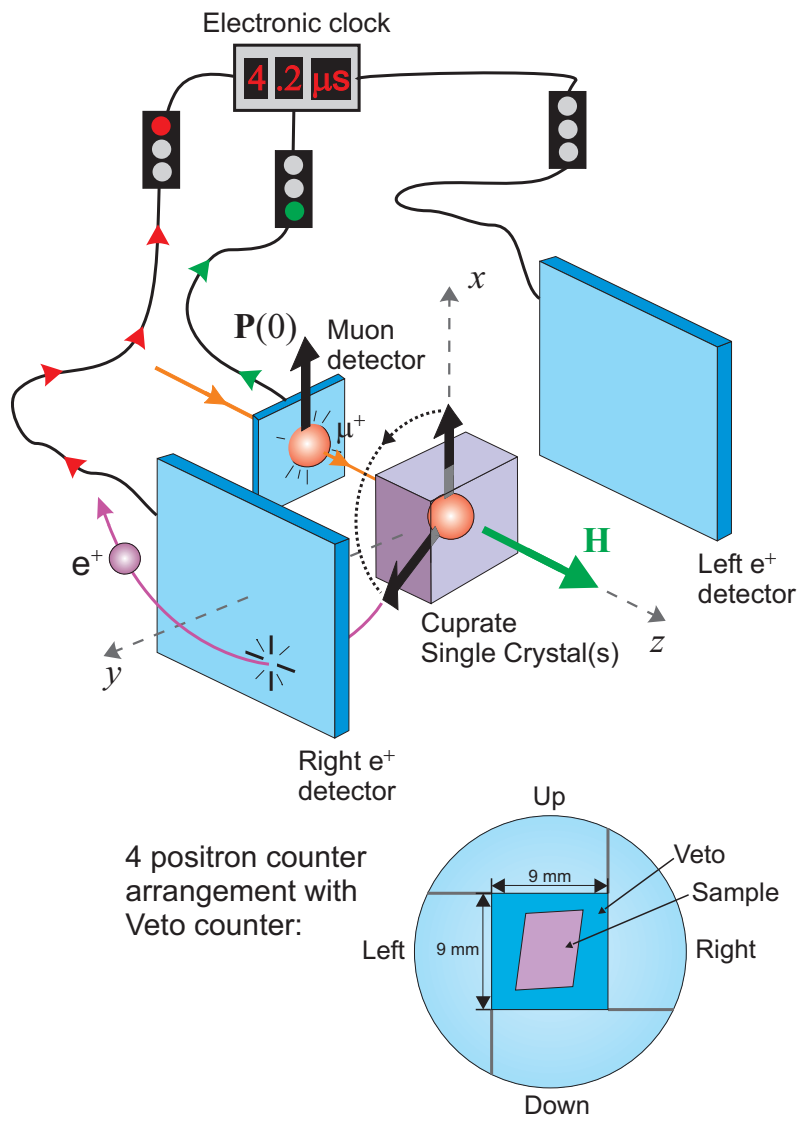

FIG. 1: (Color online) Schematic of the TF- $\mu$ SR experimental arrangement. The $\mu^{+}$beam passes through a thin plastic scintillator muon detector with its initial muon-spin polarization $\mathbf{P}(0)$ transverse to the direction of the applied magnetic field $\mathbf{H}$, which creates a start pulse for an electronic clock. The muons are subsequently implanted one-by-one in the sample, where they come to rest and Larmor precess in the local magnetic field $\mathbf{B}$. The time evolution of the muon spin polarization $P(t)$, which is affected by both static and fluctuating internal magnetic fields, is monitored via the detection of the decay positrons $\left(\mathrm{e}^{+}\right)$. The detection of a decay positron stops the electronic clock, and the corresponding elapsed-time bin of the positron-detector histogram is incremented. The positron detectors are arranged in pairs on opposite sides of the sample. In our experiments, two pairs of positron detectors surrounding the sample (Left and Right, and Up and Down) were used. In addition, the sample was mounted directly on a Veto detector, used to eliminate muons that missed the sample from contributing to the TF- $\mu \mathrm{SR}$ signal. The sample covered approximately 40 to $60 \%$ of the Veto detector (The lower figure is a depiction of the counter arrangement facing the $\mu^{+}$beam). With the exception of the incoming muon detector, all detectors were contained with the sample inside a helium-gas flow cryostat. The TF- $\mu \mathrm{SR}$ asymmetry spectrum $A(t)=a_{0} P(t)$ is formed by combining the accumulated histograms of opposing positron detectors. 

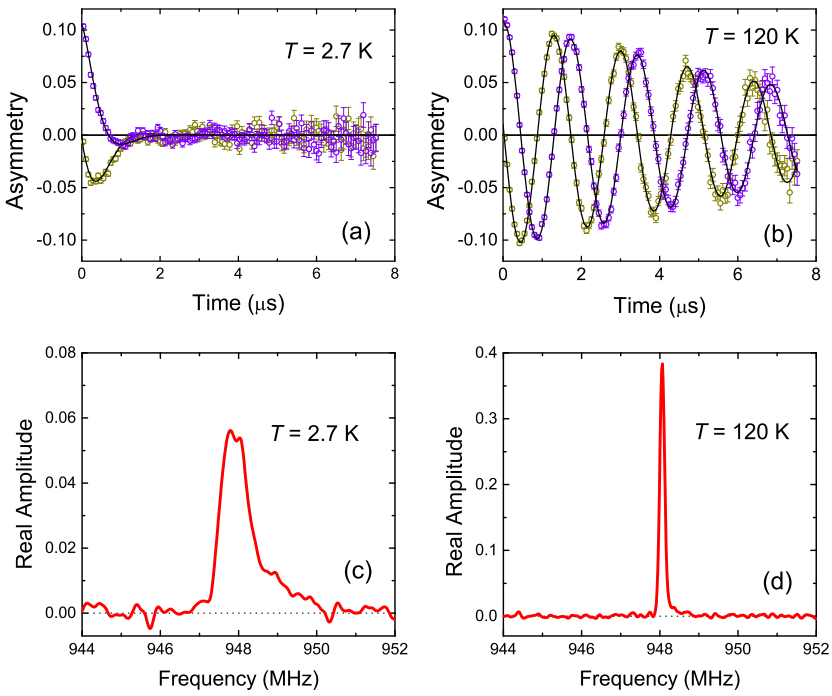

FIG. 2: (Color online) Representative TF- $\mu$ SR signals. (a) $\mathrm{TF}-\mu \mathrm{SR}$ asymmetry spectrum for optimally-doped $(p=0.165)$ YBCO at $H=7 \mathrm{~T}$ and $T=2.7 \mathrm{~K}$. The two signals, which differ in phase by $\sim 90^{\circ}$, come from the two pairs of opposing positron detectors shown in Fig. 1 (i.e. Up and Down, and Left and Right). The solid black curves through the data points are fits described in the main text. (b) Same as (a), but for $T=120 \mathrm{~K}$. (c), (d) Fourier transforms (with Gaussian apodization) of the asymmetry spectra, which provide visual depictions of the internal magnetic field distribution $n(B)$ sensed by the muon ensemble. The frequency (horizontal axis) is related to the local internal magnetic field via the relation $f=\left(\gamma_{\mu} / 2 \pi\right) B$.

tion of the decay positrons of the implanted muons.

Calibration measurements on pure $\mathrm{Ag}$ for $H=7 \mathrm{~T}$ and $H=0.5 \mathrm{~T}$ show a temperature-independent TF- $\mu \mathrm{SR}$ signal. Measurements on the cuprate samples at temperatures below $T_{c}$ were performed under field-cooled conditions, to generate the most uniform vortex lattice. At temperatures above $T_{c}$, the TF- $\mu \mathrm{SR}$ signal for all samples was found to be independent of whether the measurements were recorded under field-cooled or zero-field cooled conditions. Typical TF- $\mu \mathrm{SR}$ signals for $\mathrm{YBa}_{2} \mathrm{Cu}_{3} \mathrm{O}_{6.93}$ are presented in Fig. 2. For $T<T_{c}$ the relaxation of the TF- $\mu \mathrm{SR}$ signal is dominated by the spatial field-inhomogeneity created by the vortex lattice, which results in a broad asymmetric internal magnetic field distribution $n(B)$. Conversely, the reduced relaxation observed for $T>T_{c}$ is associated with a narrow and symmetric $n(B)$.

\section{DATA ANALYSIS AND RESULTS}

The TF- $\mu$ SR spectra were fit to

$$
A(t)=a_{0} P(t)=a_{0} G(t) \cos \left(\omega_{\mu} t+\phi\right),
$$

where $a_{0}$ is the amplitude, $\phi$ is the phase angle between the axis of the positron detector and the initial muonspin polarization $\mathbf{P}(0), \omega_{\mu}$ is the Larmor frequency and $G(t)$ is a function that describes the relaxation of the TF- $\mu$ SR signal. In particular, $G(t)=G_{\text {nuc }}(t) G_{\text {other }}(t)$, where $G_{\text {nuc }}(t)$ is a temperature-independent function due to the distribution of random nuclear dipole fields, and $G_{\text {other }}(t)$ is a phenomenological function describing the signal relaxation by other internal sources. For YBCO and BSCCO, $G_{\text {nuc }}(t)=\exp \left(-\Delta^{2} t^{2}\right)$, as is usually the case for a dense system of randomly oriented magnetic moments. However, recent ZF- $\mu$ SR measurements show that the nuclear contribution for LSCO deviates somewhat from a pure Gaussian function, $\stackrel{23}{2}$ and the relaxation of the TF- $\mu \mathrm{SR}$ signal at $T=200 \mathrm{~K}$ has the functional form $G(t)=\exp \left[-(\Lambda t)^{\beta}\right]$, with $1.72 \leq \beta \leq 1.87 \underline{\underline{20}}$ Consequently, we assume $G_{\text {nuc }}(t)=\exp (-\lambda t)^{n}$ for LSCO, where $\lambda$ and $n$ are the values of $\Lambda$ and $\beta$ determined at $T=200 \mathrm{~K}$ in Ref. 20.

For temperatures $T<0.5 T_{c}$, the internal magnetic field distribution $n(B)$ for all samples is asymmetric due to an arrangement of vortices, and satisfactory fits of the TF- $\mu \mathrm{SR}$ signal were achieved with $G_{\text {other }}(t)=\exp \left[-(\Lambda t)^{\beta}\right]$, where $1.1 \leq \beta \leq 1.8$. However, at higher temperatures $n(B)$ is symmetric, and $G_{\text {other }}(t)$ is a pure exponential relaxation function, i.e. $G_{\text {other }}(t)=\exp (-\Lambda t)$. Hence we fit the TF- $\mu$ SR signal for all samples above $T_{c}$ to

$$
A(t)=a_{0} G_{\mathrm{nuc}}(t) \exp (-\Lambda t) \cos \left(\omega_{\mu} t+\phi\right) .
$$

Figure 3 shows representative data for the temperature dependence of $\Lambda$. Below $T_{c}$ the dominant contribution to $\Lambda$ is the spatially inhomogeneous field created by vortices, which depends on the inverse square of the in-plane magnetic penetration depth $\lambda_{a b}$, the anisotropy, and the spatial arrangement of vortices. As shown in Fig. 4(a), the hole-doping dependence of $\Lambda$ for YBCO and BSCCO at low temperatures resembles the doping dependence of $\lambda_{a b}^{-2} \stackrel{24,25}{2}$ The value of $\Lambda$ is significantly smaller for BSCCO, partly because of extreme anisotropy that permits significant wandering of the vortex lines along their length $\underline{26}$

For LSCO the observed decrease of $\Lambda$ with increased hole-doping in the range $0.145 \leq p \leq 0.176$ [Fig. 4(a)] is opposite to the behavior of $\lambda_{a b}^{-2}(p) \stackrel{27}{\underline{2}}$ This is explained by a recent small-angle neutron scattering study of LSCO showing enhanced vortex-lattice disorder below $p \sim 0.18$, near a state of coexisting superconductivity and SDW order $\stackrel{28}{ }$ Such random frozen disorder of the rigid vortex lines in LSCO broadens $n(B)$ and enhances $\Lambda$. The upturn of $\Lambda$ at higher doping also opposes the behavior of $\lambda_{a b}^{-2}(p)$, which decreases beyond $p \sim 0.21,27$ This is due to Curie-like paramagnetism that dominates $\Lambda$ in heavily-overdoped LSCO, but is also present in the underdoped regime. 19,20 This contribution is evident in the temperature dependence of $\Lambda$ for optimally-doped LSCO presented in Fig. 3 $(\mathrm{g})$. There is a similar Curie term discernible in the bulk magnetic susceptibility of 

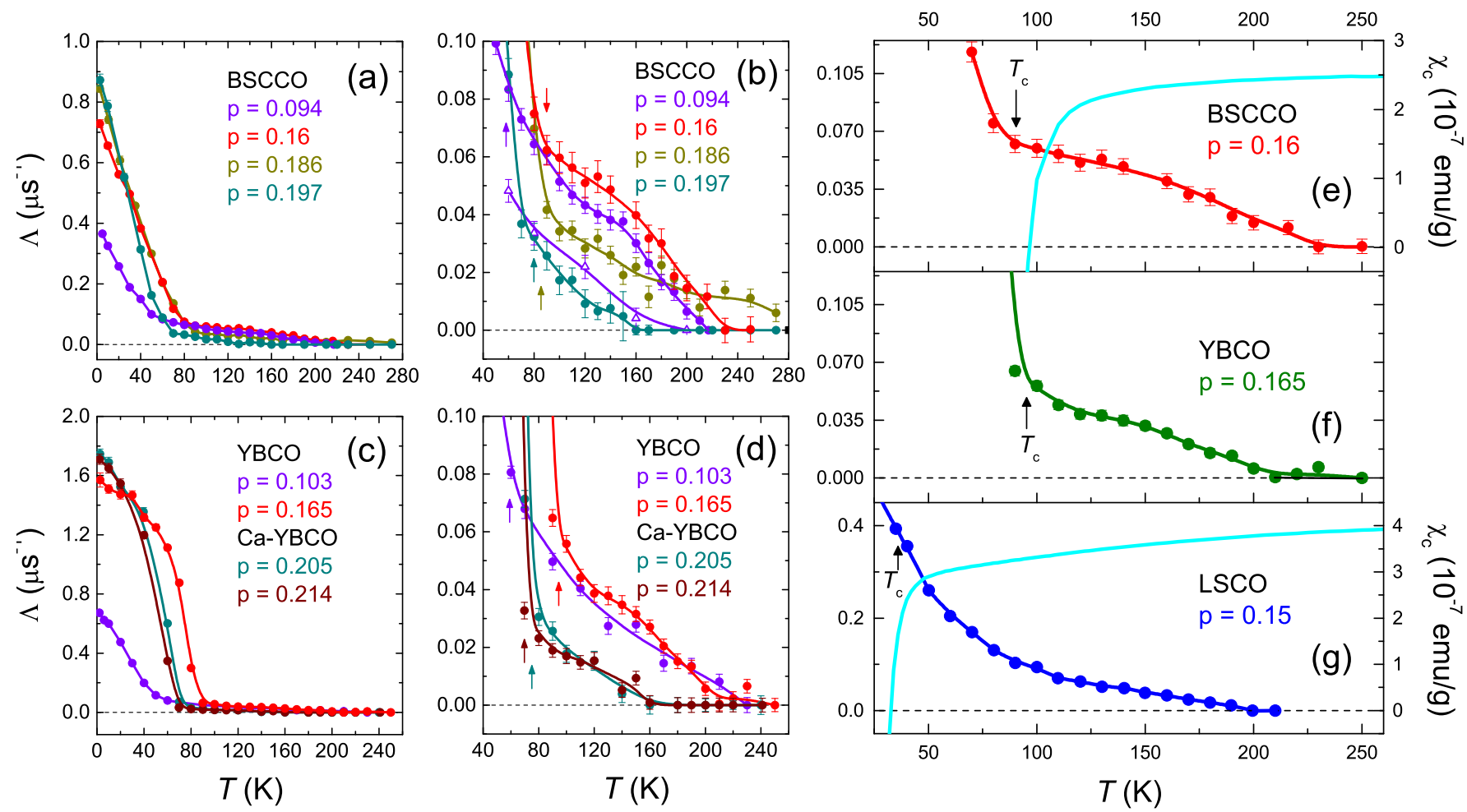

FIG. 3: (Color online) (a) Temperature dependence of $\Lambda$ at $H=7 \mathrm{~T}$ (solid circles) for BSCCO. (b) Blow-up of the hightemperature data from (a). Also shown is data for the $p=0.094$ sample at $H=0.5 \mathrm{~T}$ (open purple triangles). The arrows indicate the zero-field values of $T_{c}$ determined by bulk magnetic susceptibility. (c), (d) Representative results for pure and Ca-doped YBCO for $H=7$ T. The data at $p=0.103$ is from Ref. 18. (e), (f), (g) High-temperature behavior of $\Lambda$ (solid circles) for optimally-doped BSCCO, YBCO, and LSCO. Also shown is the bulk magnetic susceptibility (light-blue curves) for the BSCCO and LSCO samples for $H=7 \mathrm{~T}$ applied parallel to the $c$ axis.

the $p=0.197$ BSCCO sample (see Fig. [5), which may contribute somewhat to $\Lambda(T)$ at this doping. The bulk magnetic susceptibility of our BSCCO samples at $H=7 \mathrm{~T}$ resembles previous high-field measurements. $\frac{29}{2}$ This includes the Curie-like contribution to the $p=0.197$ sample, which is a common feature of heavily-overdoped cuprates.

Shifting attention to the normal state, Fig. 4(b) shows a comparison of the hole-doping dependence of $\Lambda$ at $T=1.3 T_{c}^{\max }$. Despite BSCCO possessing a higher degree of chemical disorder than YBCO, there is good agreement between the data sets for these two compounds.

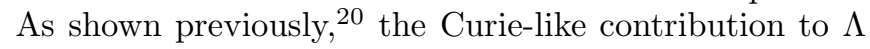
for LSCO exhibits a dominant $p$-linear dependence above $T_{c}$, with a slope $(d \Lambda / d p)$ that weakens with increasing $T$. Measurements at higher temperature show that the $p$ linear contribution extends to lower doping. Figure [6 shows the hole-doping dependence of $\Lambda$ for LSCO above $T_{c}$ (solid red circles) obtained from fits of the TF- $\mu \mathrm{SR}$ asymmetry spectra of Ref. 20 to Eq. (2). Here we stress that the values of $\Lambda$ differ from those in Ref. 20 due to the removal of the nuclear dipole contribution to $G(t)$, which must be done to compare the residual relaxation rate to the values for $\mathrm{YBCO}$ and $\mathrm{BSCCO}$. The linear growth of $\Lambda$ above $p=0.19$ at $T=40 \mathrm{~K}$ is visible below $p=0.19$ at high temperatures, and arises from the Curielike paramagnetism $\stackrel{19,20}{=}$ Subtracting this linear contribution from the hole-doping dependence of $\Lambda$ at each temperature (yielding the open red circles in Fig. (6) results in a collapse of the $\Lambda$ data for LSCO onto a universal curve shared with BSCCO and YBCO (Fig. 7). Note that for the range of comparison $0.145 \leq p \leq 0.176$, the applied field of $7 \mathrm{~T}$ corresponds to approximately one tenth of the upper critical field $H_{c 2}$ for all three compounds $\underline{30,31}$ The universal behavior for $\Lambda$ suggests that $T_{c}$ and the source of the inhomogeneous magnetic-field response above $T_{c}$ are controlled by the same energy scale.

\section{DISCUSSION}

It may be surprising to some that a significant inhomogeneous magnetic response occurs in YBCO above $T_{c}$, given that the mean free path of optimally-doped YBCO single crystals has been reported to be as large as $4 \mu \mathrm{m}{ }^{32}$ 


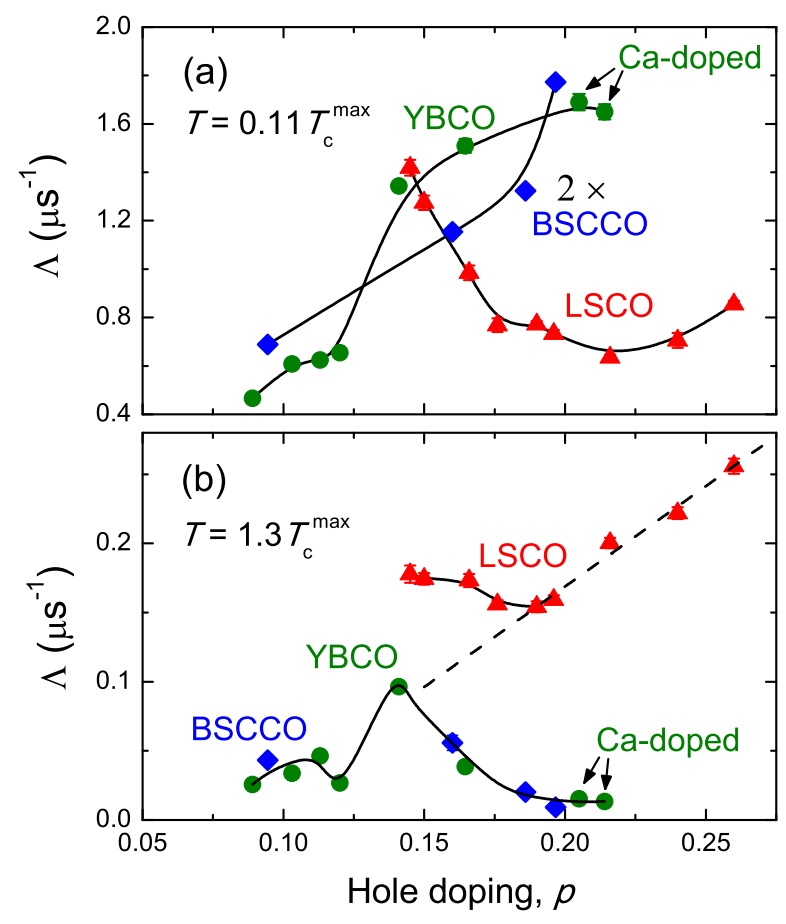

FIG. 4: (Color online) (a) Doping dependence of $\Lambda$ for $H=$ $7 \mathrm{~T}$ and $T=0.11 T_{c}^{\max }$, where $T_{c}^{\max }=90,94.1$ and $38 \mathrm{~K}$, for $\mathrm{BSCCO}, \mathrm{YBCO}$, and $\mathrm{LSCO}$, respectively. The YBCO data for $p \leq 0.141$ are from Ref. 18. For visual purposes the relaxation rate for $\mathrm{BSCCO}$ has been multiplied by a factor of 2. (b) Results for $T=1.3 T_{c}^{\max }$, where $\Lambda$ is a pure exponential relaxation rate. The BSCCO (blue diamonds) and YBCO (green circles) data are for $T=120 \mathrm{~K}$, and the LSCO data (solid red triangles) for $T=50 \mathrm{~K}$. Unlike in (a), the BSCCO data is not rescaled. The dashed line is a linear fit of the LSCO data for $p \geq 0.19$, which describes the Curie-like contribution.

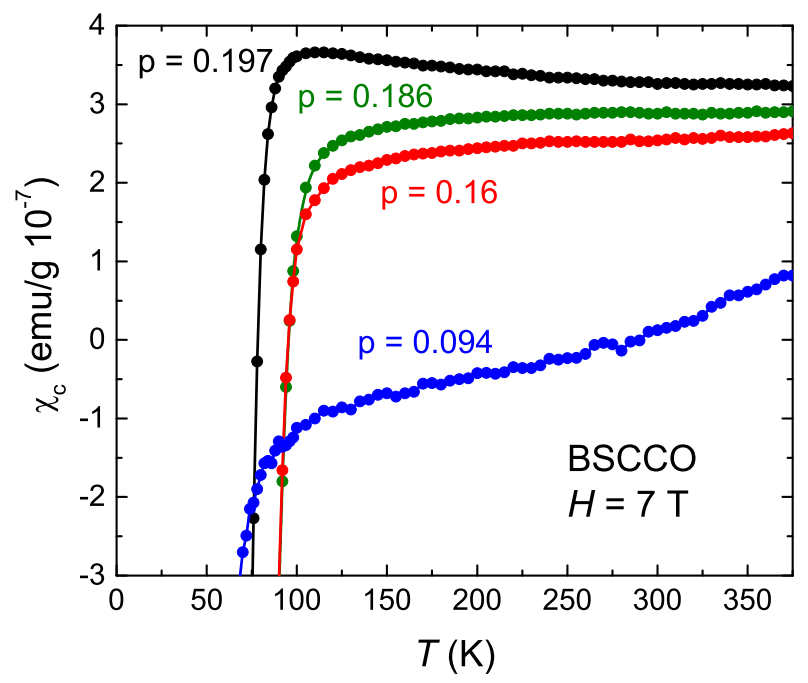

FIG. 5: (Color online) Temperature dependence of the bulk DC magnetic susceptibility of the $p=0.094,0.16,0.186$, and 0.197 BSCCO single crystals for an external magnetic field $H=7 \mathrm{~T}$ applied parallel to the $c$-axis.

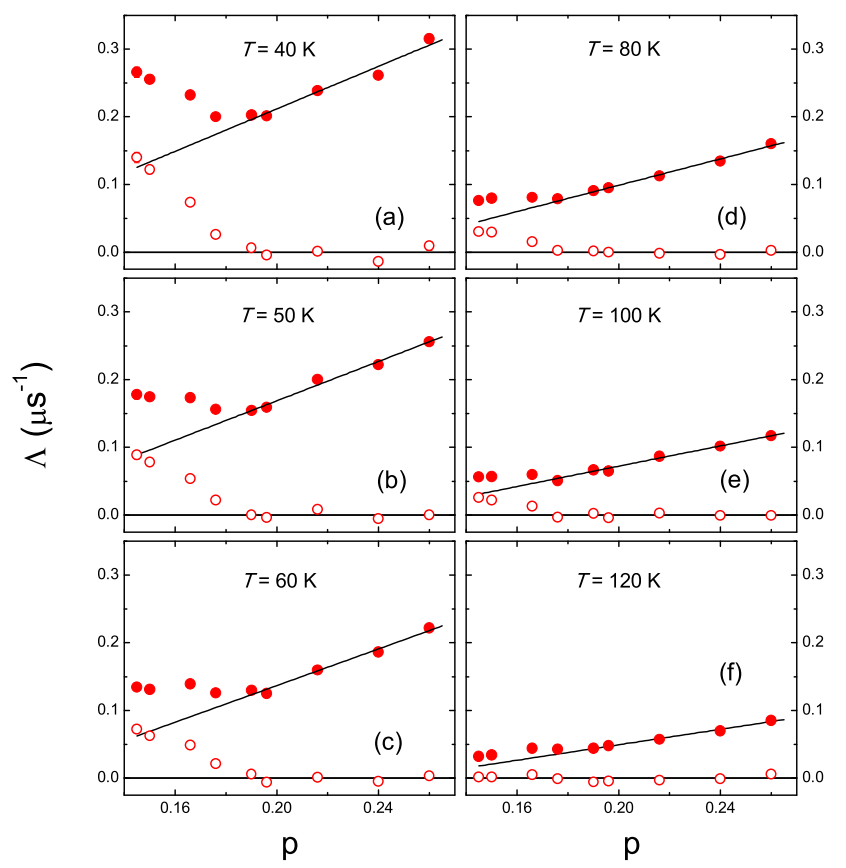

FIG. 6: (Color online) Hole-doping dependence of the exponential relaxation rate $\Lambda$ for LSCO from fits to Eq. (2) (solid red circles). The solid black line in each panel is the best fit of a straight line to the data at $p \geq 0.19$. Subtracting the straight line fit from $\Lambda$ yields the open red circles.

However, this estimate is at low temperatures far below $T_{c}$ and in zero applied magnetic field. One of the reasons that the mean free path is so long at low temperatures is that the superconducting gap reduces the available phase space for impurity scattering. ${ }^{33}$ In fact there is a dramatic increase of the scattering rate of optimally-doped YBCO above $20 \mathrm{~K}, 32$ indicating a large reduction of the mean free path with increasing temperature well before the normal state is reached. In addition, experiments observing quantum oscillations in the electrical resistance of underdoped YBCO indicate a significant reduction of the mean free path in the presence of an applied magnetic field ${ }^{34}$ For example, at $35 \mathrm{~T}$, the mean free path of $\mathrm{YBa}_{2} \mathrm{Cu}_{3} \mathrm{O}_{6.5}$ at low temperatures is roughly estimated to be only $0.016 \mu \mathrm{m}^{35}$

Previous measurements on YBCO revealed a clear reduction of $\Lambda$ near $p=0.12, \underline{18}$ where recent neutron scattering measurements for $H=0$ show enhanced incommensurate CDW correlations,,$\frac{4}{,}$ and nuclear magnetic resonance experiments at $H=28.5 \mathrm{~T}$ show static commensurate CDW order ${ }^{36}$ Enhanced incommensurate SDW order is also observed in LSCO near $p=0.12 \stackrel{37}{*}$ However, slowing down of charge or spin fluctuations should increase the value of $\Lambda$. Moreover, while $\Lambda$ above $T_{c}$ increases with $H$ [Fig. 3(b)], even fields well in excess of $7 \mathrm{~T}$ have no effect on CDW or SDW fluctuations above $T_{c}, \frac{5,38}{4}$ Hence CDW or SDW correlations, which are confined to a narrow range of doping and seem to compete with superconductivity, cannot be the primary source of the resid- 


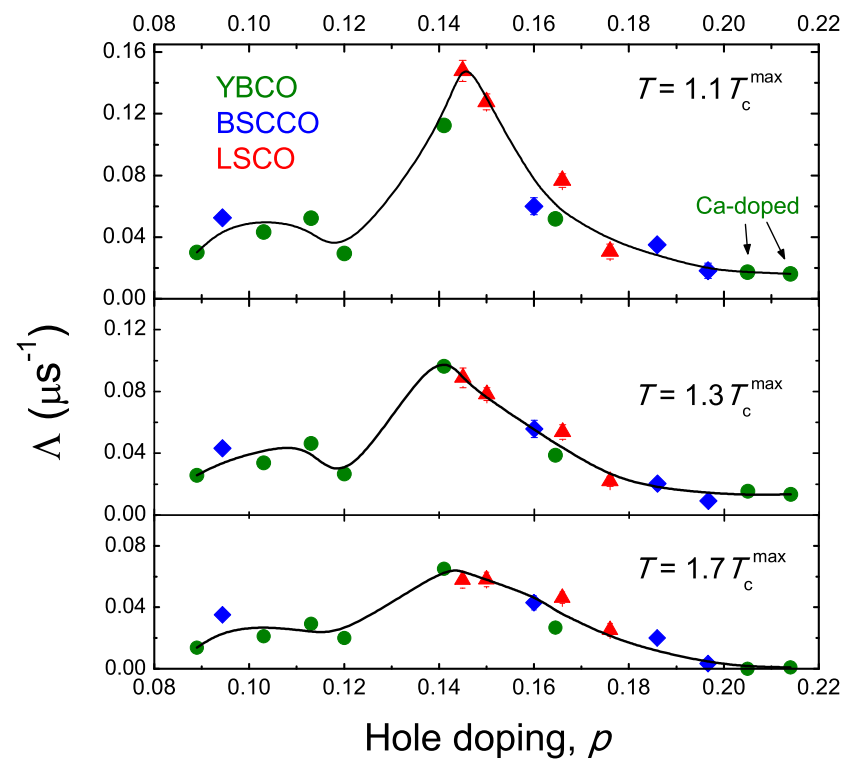

FIG. 7: (Color online) Doping dependence of $\Lambda$ for $T=1.1$, 1.3 and $1.7 T_{c}^{\max }$. The $p$-linear dependent contribution to the data for LSCO has been subtracted, as described in the main text. The solid curves are guides to the eye.

ual inhomogeneous line broadening shown in Fig. 7 that is observed over the wide doping range $0.089<p<0.214$. Additionally, we point out that the muon has no electric quadrupole moment and hence does not sense CDW order directly. What it can detect is modifications of the second moment of the distribution of nuclear dipolar magnetic fields by quadrupole interactions of the host nuclei with the local electric-field gradient (EFG) asociated with CDW order. But this is only true in zero or weak applied magnetic field. Although the dipolar fields of the nuclei are dependent on both their electric and magnetic interactions, $\stackrel{39}{=}$ when the applied magnetic field is strong (as in our experiments), the external field direction becomes a natural quantization axis for the nuclear spin system. In this case the second moment of the nuclear dipolar field distribution is independent of the magnitude of the external field and is not altered by changes in the local EFG.

Figure 8 shows that $\Lambda$ persists above the pseudogap temperature $T^{*}$ at high doping, but vanishes below $T^{*}$ in the underdoped region, indicating an origin not solely related to the pseudogap phase. Instead the doping dependence and universal scaling of $\Lambda$ with $T_{c}^{\max }$ suggest that the inhomogenous field response above $T_{c}$ is associated with superconducting correlations. Like the vortex Nernst signal ${ }^{9}$ and diamagnetism observed by torque magnetometry $\stackrel{11.12}{\Perp} \Lambda$ for $T>T_{c}$ is reduced with increasing temperature and enhanced by the external field. The diamagnetism and vortex Nernst signal have been attributed to SCFs, which are observed over a narrow range of temperature above $T_{c}$ on a frequency scale of $0.01-10 \mathrm{THz}: \frac{8,16,17}{2}$ At somewhat higher temperatures,
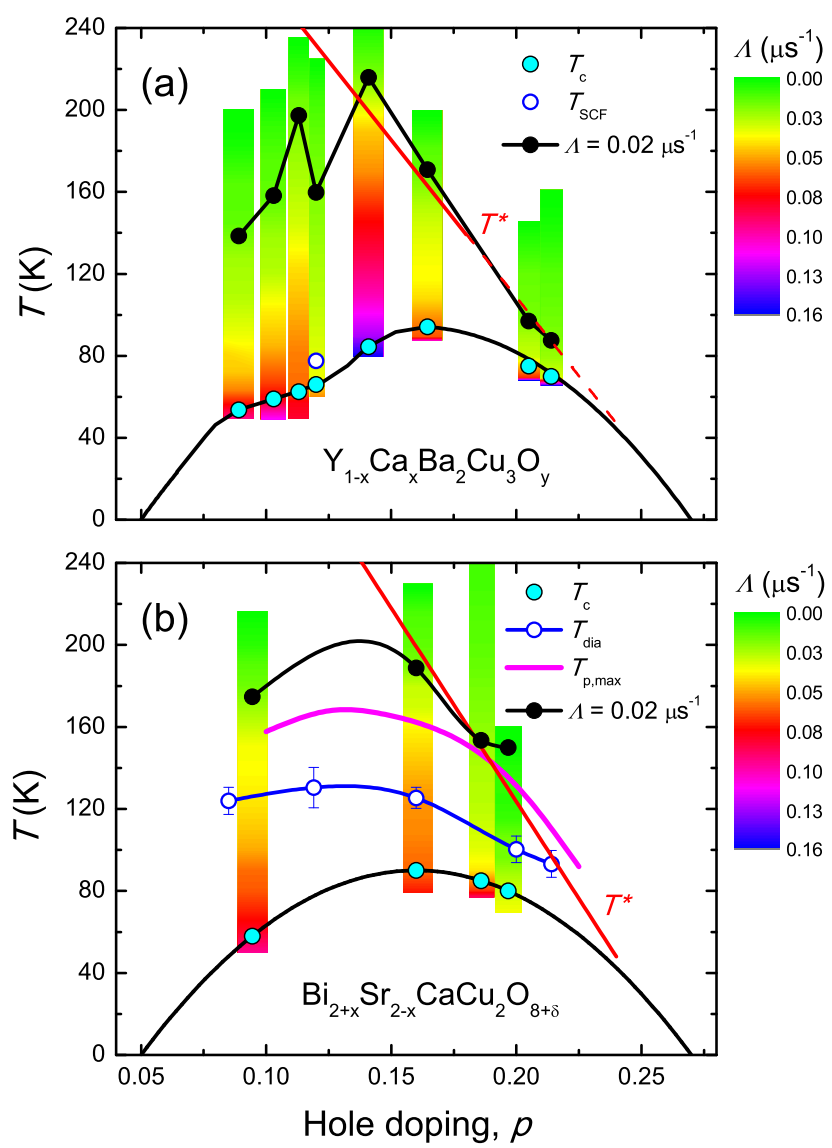

FIG. 8: (Color online) (a) Contour bar graph of the variation of $\Lambda$ with temperature and hole doping in pure and Ca-doped YBCO for $H=7 \mathrm{~T}$, achieved by interpolation of the $\Lambda$ vs. $T$ data sets. The black connected circles indicate the arbitrary value $\Lambda=0.02 \mu \mathrm{s}^{-1}$. Also shown is the onset temperature $T_{\mathrm{SCF}}$ for SCFs at $p=0.12$ inferred from Nernst-effect measurements, and $T^{*}$ (solid red line) extrapolated to higher doping (dashed red line) from Ref. 2. (b) Similar schematic phase diagram for BSCCO, where $T_{\text {dia }}$ is the temperature at which torque magnetometry measurements detect the onset of diamagnetism ${ }^{11}$ and $T^{*}$ is from Ref. 41 . The $T_{p, \max }$ curve indicates the temperature above which STM measurements ${ }^{10}$ on BSCCO find less than $10 \%$ of the sample containing nanometer-sized regions with pairing gaps.

our experiments show $\Lambda$ varying on the order of 0.01 $0.1 \mu \mathrm{s}^{-1}$ (Fig. 7). Considering for a moment $\Lambda$ to be a dynamic relaxation rate due to fast fluctuations of the local field, $\Lambda=\gamma_{\mu}^{2}\left\langle(\delta B)^{2}\right\rangle / \nu$, where $\nu$ is the fluctuation frequency and $\left\langle(\delta B)^{2}\right\rangle$ is the second moment of $n(B)$ in the static limit $(\nu \rightarrow 0)$. For $0.01 \mathrm{THz}$ fluctuations, the range $0.01<\Lambda<0.1 \mu \mathrm{s}^{-1}$ corresponds to a static line width of $0.012<\sqrt{\left\langle(\delta B)^{2}\right\rangle}<0.037 \mathrm{~T}$, which exceeds the line width of $n(B)$ associated with the frozen vortex lattice below $T_{c}$. Hence the vortex liquid inferred from the Nernst signal above $T_{c}$ is not detectable by $\mu \mathrm{SR}$. This 
conclusion is supported by a $\mu \mathrm{SR}$ study of the sizeable

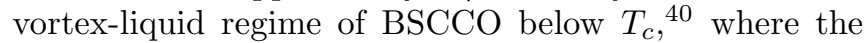
$\mathrm{TF}-\mu \mathrm{SR}$ line width is severely narrowed by thermal vortex fluctuations. In stark contrast to the field dependence of $\Lambda$ observed above $T_{c}$ [Compare the data of $p=0.094$ for $H=0.5 \mathrm{~T}$ and $H=7 \mathrm{~T}$ in Fig. [3(b)], the TF- $\mu \mathrm{SR}$ line width previously measured in the vortex-liquid phase of BSCCO is reduced by stronger applied magnetic field.

Although homogeneous SCFs do not produce $\mu \mathrm{SR}$ line broadening, spatially varying SCFs will. The local pairing observed by $\mathrm{STM}^{10}$ on BSCCO above $T_{c}$ is characterized by a distribution of gap sizes and a partial suppression of the density of states at the Fermi energy $N\left(E_{F}\right)$, which vanish inhomogeneously with increasing $T$. In a conventional metal the Pauli susceptibility $\chi_{0}$ associated with the conduction electrons is proportional to $N\left(E_{F}\right)$. Spatial variations in the magnitude of $\chi_{0}$ associated with nanoscale regions of pairing cause inhomogeneous broadening via the hyperfine coupling between the $\mu^{+}$and the spin polarization of the surrounding conduction electrons. Yet any such contribution to $\Lambda$ must be minor, since above $T_{c}$ the depletion of $N\left(E_{F}\right)$ in the underdoped regime is dominated by the spatially inhomogeneous pseudogap.

Regular inhomogeneous regions of SCFs may occur in the bulk from a competition with some other kind of fragile order. One candidate is fluctuating stripes, which are characterized by a dynamical unidirectional modulation of charge, or both spin and charge $\stackrel{42}{*}$ In this environment inhomogeneous line broadening will result from muons experiencing distinct time-averaged local fields $\langle B(t)\rangle$ in different parts of the sample. Such will be the case for muons stopping inside regions with different strengths of fluctuation diamagnetism. However, even if the regions in which SCFs persist are comparable throughout the sample, inhomogeneous line broadening results from muons stopping in intermediate or surrounding areas. These muons experience the expelled time-averaged field, which diminishes in magnitude with increased stopping distance away from the diamagnetic regions. As shown in Fig. 8 (b), $\Lambda$ tracks the pairing gap coverage in BSCCO observed by STM - where the latter is presumably similar regardless of whether the regions of SCFs maintain a regular pattern or are broken up into irregular-shaped patches by disorder.

While our findings strongly favor an interpretation involving inhomogeneous SCFs, current instrument detection limits prevent us from determining whether the magnetic response above $T_{c}$ is diamagnetic. Even so, our results show that there is an intrinsic electronic propensity toward inhomogeneity in the normal-state of high- $T_{c}$ cuprate superconductors, where even weak disorder of the kind found in $\mathrm{YBCO}$ is sufficient to spatially pin the non-uniform electron liquid.

We gratefully acknowledge informative and insightful discussions with S.A. Kivelson, W.N. Hardy, A. Yazdani and L. Taillefer. This work was supported by the Natural Sciences and Engineering Research Council of Canada, the Canadian Foundation for Innovation, the Canadian Institute for Advanced Research, and $\mathrm{CNPq} /$ Brazil.
* Current Address: Department of Physics, Simon Fraser University, Burnaby, British Columbia, Canada V5A 1S6

1 V. Hinkov, D. Haug, B. Fauqué, P. Bourges, Y. Sidis, A. Ivanov, C. Bernhard, C.T. Lin, and B. Keimer, Science 319, 597-600 (2008).

2 R. Daou, J. Chang, D. LeBoeuf, O. Cyr-Choinière, F. Laliberté, N. Doiron-Leyraud, B.J. Ramshaw, R. Liang, D.A. Bonn, W.N. Hardy, and L. Taillefer, Nature 463, 519-522 (2010).

3 C.V. Parker, P. Aynajian, E.H. da Silva Neto, A. Pushp, S. Ono, J. Wen, Z. Xu, G. Gu, and A. Yazdani, Nature 468, 677-680 (2010).

4 G. Ghiringhelli, M. Le Tacon, M. Minola, S. BlancoCanosa, C. Mazzoli, N.B. Brookes, G.M. De Luca, A. Frano, D.G. Hawthorn, F. He, T. Loew, M. Moretti Sala, D.C. Peets, M. Salluzzo, R. Sutarto, G.A. Sawatzky, E. Weschke, B. Keimer, and L. Braicovich, Science 337, 821825 (2012).

5 J. Chang, E. Blackburn, A.T. Holmes, N.B. Christensen, J. Larsen, J. Mesot, R. Liang, D.A. Bonn, W.N. Hardy, A. Watenphul, M.V. Zimmermann, E.M. Forgan, and S.M. Hayden, Nature Physics 8, 871-876 (2012).

6 B. Fauqué, Y. Sidis, V. Hinkov, S. Pailhès, C.T. Lin, X. Chaud, and P. Bourges, Phys. Rev. Lett. 96, 197001 (2006).

7 Y. Li, V. Balédent, N. Barišić, Y. Cho, B. Fauqué, Y. Sidis, G. Yu, X. Zhao, P. Bourges, and M. Greven, Nature 455,
372-375 (2008).

8 J. Corson, R. Mallozzi, J. Orenstein, J. N. Eckstein, and I. Bozovic, Nature 398, 221-223 (1999).

9 Z.A. Xu, N.P. Ong, Y. Wang, T. Kakeshita, and S. Uchida, Nature 406, 486-488 (2000).

10 K.K. Gomes, A.N. Pasupathy, A. Pushp, S. Ono, Y. Ando, and A. Yazdani, Nature 447, 569-572 (2007).

11 Y. Wang, L. Li, M.J. Naughton, G.D. Gu, S. Uchida, N.P. Ong, Phys. Rev. Lett. 95, 247002 (2005).

12 L. Li, Y. Wang, S. Komiya, S. Ono, Y. Ando, G.D. Gu, and N.P. Ong, Phys. Rev. B 81, 054510 (2010).

13 A. Dubroka, M. Rössle, K.W. Kim, V.K. Malik, D. Munzar, D.N. Basov, A.A. Schafgans, S.J. Moon, C.T. Lin, D. Haug, V. Hinkov, B. Keimer, Th. Wolf, J.G. Storey, J.L. Tallon, and C. Bernhard, Phys. Rev. Lett. 106, 047006 (2011).

14 O. Cyr-Choinière, R. Daou, F. Laliberté, D. LeBoeuf, N. Doiron-Leyraud, J. Chang, J.-Q. Yan, J.-G. Cheng, J.-S. Zhou, J.B. Goodenough, S. Pyon, T. Takayama, H. Takagi, Y. Tanaka, and L. Taillefer, Nature 458, 743-745 (2009).

${ }^{15}$ F. Rullier-Albenque, R. Tourbot, H. Alloul, P. Lejay, D. Colson, and A. Forget, Phys. Rev. Lett. 96, 067002 (2006).

16 M. S. Grbić, M. Požek, D. Paar, V. Hinkov, M. Raichle, D. Haug, B. Keimer, N. Barišić, and A. Dulčić, Phys. Rev. B 83, 144508 (2011).

17 L.S. Bilbro, R. Valdés Aguilar, G. Logvenov, O. Pelleg, I. Božović, and N.P. Armitage, Nature Physics 7, 298-302 
(2011)

18 J. E. Sonier, M. Ilton, V. Pacradouni, C. V. Kaiser, S. A. Sabok-Sayr, Y. Ando, S. Komiya, W. N. Hardy, D. A. Bonn, R. Liang, and W. A. Atkinson, Phys. Rev. Lett. 101, 117001 (2008).

19 G. J. MacDougall, A. T. Savici, A. A. Aczel, R. J. Birgeneau, H. Kim, S.-J. Kim, T. Ito, J. A. Rodriguez, P. L. Russo, Y. J. Uemura, S. Wakimoto, C. R. Wiebe, and G. M. Luke, Phys. Rev. B 81, 014508 (2010).

20 C.V. Kaiser, W. Huang, S. Komiya, N.E. Hussey, T. Adachi, Y. Tanabe, Y. Koike, and J.E. Sonier, Phys. Rev. B 86, 054522 (2012).

21 R. Liang, D.A. Bonn, and W.N. Hardy, Physica C 304, 105-111 (1998).

22 J.E. Sonier, V. Pacradouni, S.A. Sabok-Sayr, W.N. Hardy, D.A. Bonn, R. Liang, and H.A. Mook, Phys. Rev. Lett. 103, 167002 (2009).

23 W. Huang, V. Pacradouni, M.P. Kennett, S. Komiya, and J.E. Sonier, Phys. Rev. B 85, 104527 (2012).

24 J.E. Sonier, S.A. Sabok-Sayr, F.D. Callaghan, C.V. Kaiser, V. Pacradouni, J.H. Brewer, S.L. Stubbs, W.N. Hardy, D.A. Bonn, R. Liang, and W.A. Atkinson, Phys. Rev. B 76, 134518 (2007).

25 W. Anukool, S. Barakat, C. Panagopoulos, and J.R. Cooper, Phys. Rev. B 80, 024516 (2009).

${ }^{26}$ E.H. Brandt, Phys. Rev. Lett. 66, 3213 (1991).

27 T.R. Lemberger, I. Hetel, A. Tsukada, M. Naito, and M. Randeria, Phys. Rev. B 83, 140507(R) (2011).

28 J. Chang, J.S. White, M. Laver, C.J. Bowell, S.P. Brown, A.T. Holmes, L. Maechler, S. Strässle, R. Gilardi, S. Gerber, T. Kurosawa, N. Momono, M. Oda, M. Ido, O.J. Lipscombe, S.M. Hayden, C.D. Dewhurst, R. Vavrin, J. Gavilano, J. Kohlbrecher, E.M. Forgan, and J. Mesot, Phys. Rev. B 85, 134520 (2012).

29 T. Watanabe, T. Fujii, and A. Matsuda, Phys. Rev. Lett. 84, 5848-5851 (2000).

30 P.M.C Rourke, I. Mouzopoulou, X. Xu, C. Panagopoulos, Y. Wang, B. Vignolle, C. Proust, E.V. Kurganova, U. Zeitler, Y. Tanabe, T. Adachi, Y. Koike, and N.E. Hussey, Nature Physics 7, 455-458 (2011).
31 G. Grissonnanche, O. Cyr-Choinière, F. Laliberté, S. René de Cotret, A. Juneau-Fecteau, S. Dufour-Beauséjour, M.È. Delage, D. LeBoeuf, J. Chang, B.J. Ramshaw, D.A. Bonn, W.N. Hardy, R. Liang, S. Adachi, N.E. Hussey, B. Vignolle, C. Proust, M. Sutherland, S. Krämer, J.H. Park, D. Graf, N. Doiron-Leyraud, and L. Taillefer, arXiv:1303.3856.

32 A. Hosseini, R. Harris, S. Kamal, P. Dosanjh, J. Preston, R. Liang, W.N. Hardy, and D.A. Bonn, Phys. Rev. B 60, 1349-1359 (1999).

33 T.S. Nunner, and P.J. Hirschfeld, Phys. Rev. B 72, 014514 (2005).

34 N. Doiron-Leyraud, C. Proust, D. LeBoeuf, J. Levallois, J.-B. Bonnemaisson, R. Liang, D.A. Bonn, W.N. Hardy, and L. Taillefer, Nature 447, 565-568 (2007).

35 C. Jaudet, J. Levallois, A. Andouard, D. Vignolles, B. Vignolle, R. Liang, D.A. Bonn, W.N. Hardy, N.E. Hussey, L. Taillefer, and C. Proust, Physica B 404 354-356 (2009).

36 T. Wu, H. Mayaffre, S. Krämer, M. Horvatić, C. Berthier, W.N. Hardy, R. Liang, D.A. Bonn, and M.H. Julien, Nature 477, 191-194 (2011).

37 S. Wakimoto, R.J. Birgeneau, Y.S. Lee, and G. Shirane, Phys. Rev. B 63, 172501 (2001).

38 B. Lake, G. Aeppli, K.N. Clausen, D.F. McMorrow, K. Lefmann, N.E. Hussey, N. Mangkorntong, M. Nohara, H. Takagi, T.E. Mason, and A. Schröder, Science 291, 17591762 (2001).

39 O. Hartmann, Phys. Rev. Lett. 39, 832-835 (1977).

40 S.L. Lee, M. Warden, H. Keller, J.W. Schneider, D. Zech, P. Zimmermann, R. Cubitt, E.M. Forgan, M.T. Wylie, P.H. Kes, T.W. Li, A.A. Menovsky, and Z. Tarnawski, Phys. Rev. Lett. 75, 922-925 (1995).

41 J.K. Ren, X.B. Zhu, H.F. Yu, Y. Tian, H.F. Yang, C.Z. Gu, N.L. Wang, Y.F. Ren, and S.P. Zhao, Scientific Reports 2, 248 (2012).

42 S.A. Kivelson, I.P. Bindloss, E. Fradkin, V. Organesyan, J.M. Tranquada, A. Kapitulnik, and C. Howard, Rev. Mod. Phys. 75, 1201-1241 (2003). 